\title{
PNA-mediated Real-Time PCR Clamping for Detection of EGFR Mutations
}

\author{
Jae-jin Choi, Minhey Cho, Miae Oh, Hyunsun Kim, Min-seock Kil, and Heekyung Park* \\ Panagene Inc., 816, Tamnip-Dong, Youseong-Gu, Daejeon 305-510, Korea. *E-mail: hkpark@panagene.com \\ Received August 26, 2010, Accepted September 16, 2010
}

\begin{abstract}
Tyrosine kinase inhibitors (TKIs) are currently used in the treatment of patients with advanced lung cancer. Recent studies on non-small cell lung cancer have shown that some patients carry somatic mutations in the epidermal growth factor receptor (EGFR) gene. Such mutations correlate with the effectiveness of certain TKIs. To detect a small amount of mutant EGFR among an abundance of wild-type EGFR, we have developed a highly sensitive and simple method using PNAmediated real-time PCR clamping. The PNA-mediated real-time PCR clamping enables detection of EGFR mutants down to approximately $1 \%$ mutant -to- wild type. The total assay time was short as it required only $2.0 \mathrm{hr}$. Thus, PNAmediated real-time PCR clamping can easily be applied to clinical samples for identification of DNA carrying EGFR mutations and also appear to be the best assay to detect somatic mutations.
\end{abstract}

Key Words: EGFR, Mutation, PNA, Cancer, PCR clamping

\section{Introduction}

Tyrosine kinase inhibitors (TKIs) have shown antitumor activities in patients with advanced Non-small cell lung cancers (NSCLCs). ${ }^{1-3}$ Many researchers have found associations between the presence of somatic epidermal growth factor receptor (EGFR) mutations. EGFR mutant detection in tumor tissue have been used to predict responses to TKI treatment, and thus to guide treatment of patients with advanced NSCLC. ${ }^{4-5}$

Detection of EGFR mutants is usually achieved by sequencing of DNA amplified from tumor tissues. ${ }^{4-5}$ However, diagnostic sequencing techniques are excessively complex and timeconsuming when used as routine protocols, and can detect only mutant sequences that constitute $>20 \%$ of total gene content. ${ }^{6}$ This complexity is confounded by the fact that clinical samples often contain a small subpopulation of mutant cells mixed with a greater level of normal tissue, thus sometimes resulting in nondetection of mutations by sequencing technologies. ${ }^{7}$ Recently, several methods, including polymerase chain reaction-restriction fragment length polymorphism (PCR-RFLP), single-strand conformation polymorphism (PCR-SSCP), mutant-allele-specific amplification, and mutation enrichment assays, have been used to detect EGFR mutations. ${ }^{8-9}$ These methods include several steps and/or are time-consuming; thus, such approaches are impracticable for routine clinical use. ${ }^{10}$ Also, detection of an EGFR mutation in a NSCLC patient requires that a very sensitive assay should be used because the level of mutant-type EGFR DNA will be lower than that of wild-type EGFR DNA.

We have developed a highly sensitive and simple method using PNA-mediated real-time PCR clamping (PNA clamp) to detect a small amount of mutant-type EGFR DNA in the presence of an abundance of the wild-type EGFR DNA. We evaluated the specificity and sensitivity of PNA clamp, and validated its use for EGFR mutation detection on cell lines.

\section{Experimental Section}

PNA probe synthesis. PNA probes were synthesized em- ploying Panagene (Panagene Inc, Daejeon, Korea) technology, using benzothiazole-2-sulfonyl as the amine-protecting group. ${ }^{10}$ A high-performance liquid chromatograph (Agilent 1100; Agilent Technologies, Wilmington, DE) was used to purify the PNA probes, the quality of which was assessed by MALDITOF (AX1MA-CFR; Shimadzu Co, Kyoto, Japan). The concentration of each PNA probe was determined using a NanoDrop ND-1000 spectrophotometer (NanoDrop Technologies, Wilmington, DE). To permit selection of optimum PNA probes, several candidates were designed for each target.

Plasmid cloning and sequencing analysis. Wild-type DNA fragments containing exon 19, 20, and 21 of the EGFR gene (identified in UniGene Cluster entry \# Hs.77432) were amplified by PCR from normal human genomic DNA. The primers used were EGFR-exon19-F-1, 5'-GCGTACATTTGTCCTTCCAAA-3', and EGFR-exon19-R, 5'-GGCCAGTGCTGTCTCTAAGG-3', for exons19; EGFR-exon20-F, 5'-CTCTCCCACTGCATCTGTCA-3', and EGFR-exon20-R, 5'-GATGGGACAGGCACTGATTT-3', for exon 20; and EGFRexon21-F, 5'-CCTCACAGCAGGGTCTTCTC-3', and EGFRexon21-R, 5'-ATCCTGCAGGGAGAGACTGA-3', for exon 21. Amplified fragments were cloned into the pGEM-T-Easy vector (Promega, Madison, WI). DNA fragments harboring individual EGFR mutations were prepared using the cloned fragments as templates, and employing a QuikChange $\AA$ site-directed mutagenesis kit (Stratagene, La Jolla, CA); the fragments were finally inserted into the pGEM-T-Easy vector.

Cell lines and cell culture. H1975 cell line (adenocarcinoma) was obtained from the American Type of Culture Collection (Rockville, MD, USA) and PC-9, HCC827GR cell line were obtained from the Konkuk University Medical Center (Seoul, Korea). Cell lines were expanded in RPMI-1640 medium containing $10 \%(\mathrm{v} / \mathrm{v})$ fetal calf serum and cultured according to standard procedures. H1975 cells contain T790M and L858R point mutations in exon 20 and 21. PC-9 and HCC827GR cells contain the exon 19 Glu746-Ala750 deletion mutation. Genomic DNA was prepared using a High Pure PCR Template Preparation Kit (Roche Applied Science, Mannheim, Germany). 
Table 1. EGFR mutational types detected in this study

\begin{tabular}{ccc}
\hline & \multicolumn{2}{c}{ Mutation type } \\
\cline { 2 - 3 } & Codon & Nucleotide \\
\hline Exon 19 & G746_A750del & 2235_2249 del 15 \\
Exon 20 & T790M & ACG $\rightarrow$ ATG \\
Exon 21 & L858R & CTG $\rightarrow$ CGG \\
\hline
\end{tabular}

PCR reaction. Real-time PCR reactions were performed using a CFX 96 (Bio-Rad, USA). PCR cycling commenced with a 5 min hold at $94{ }^{\circ} \mathrm{C}$ followed by 40 cycles of $94{ }^{\circ} \mathrm{C}$ for $30 \mathrm{sec}$, $70{ }^{\circ} \mathrm{C}$ for $20 \mathrm{sec}, 63{ }^{\circ} \mathrm{C}$ for $30 \mathrm{sec}$, and $72{ }^{\circ} \mathrm{C}$ for $30 \mathrm{sec}$. Three EGFR mutation types were detected using PNA-mediated realtime PCR clamping in this study (Table 1). PCR efficiency was determined by measuring the threshold cycle $(\mathrm{Ct})$ value. Obtain $\mathrm{Ct}$ values for the control and mutation assays by observing the SYBRGreen amplification plots. Calculate the delta $\mathrm{Ct}(\Delta \mathrm{Ct})$ value as follows, ensuring that the sample and control $\mathrm{Ct}$ values are from the sample to analyze and wild-type control sample. [Control Ct] - [Sample Ct] $=\Delta \mathrm{Ct}$. The cut-off $\Delta \mathrm{Ct}$ was defined as 2 for the G746_A750del, T790M and L858R.

\section{Results}

Establishment of PNA mediated real-time PCR clamping. PNA-mediated real-time PCR clamping can quickly and reliably detect specific mutations or deletions at known positions. In this method, PNA probes and DNA primers are used together in the PCR clamping reaction. Positive signals are detected by intercalation of SYBRGreen fluorescent dye. The approach is schematically presented in Figure 1. The PNA probe suppresses amplification of the wild-type sequence (Fig. 1A and 1B), thereby enhancing preferential amplification of mutant sequences by competitively inhibiting DNA primer binding to wildtype DNA (Fig. 1C and 1D). PCR efficiency was determined by measuring the threshold cycle $(\mathrm{Ct})$ value. The $\mathrm{Ct}$ values of control and sample obtained by observing the SYBRGreen amplification plots (Fig. 1B and 1D).

Optimization of PNA-mediated real-time PCR clamping. Several PNA probes were designed based on point mutation was located in the center of probe. Their length and Tm values were taken into consideration. Also, taking advantage of superb base discrimination capability of PNA, we designed the reaction scheme of PNA-directed clamping PCR (Fig. 1), in which the PNA must complete effectively against its cognate PCR primer in binding to their common recognition sited. To optimize PNA annealing to the perfectly matched wild type-template, the normal three step PCR cycle was expanded with a PNA annealing step between the heat denaturation and the PCR primer annealing step (Fig. 2A). During the step at a temperature higher than the step of PCR primer annealing, only PNA will be allowed to bind to its target sequence. To optimize the PNA annealing and primer annealing conditions for PCR clamping, the effects of PNA annealing temperature $\left(67-72{ }^{\circ} \mathrm{C}\right)$ and primer annealing temperature $\left(57-65^{\circ} \mathrm{C}\right)$ on threshold cycle $(\mathrm{Ct})$ and $\Delta \mathrm{Ct}$ (Control $\mathrm{Ct}$-mutant $\mathrm{Ct}$ ) were examined. For the PNA PCR clamping reaction, there seemed to be an optimal
(A)
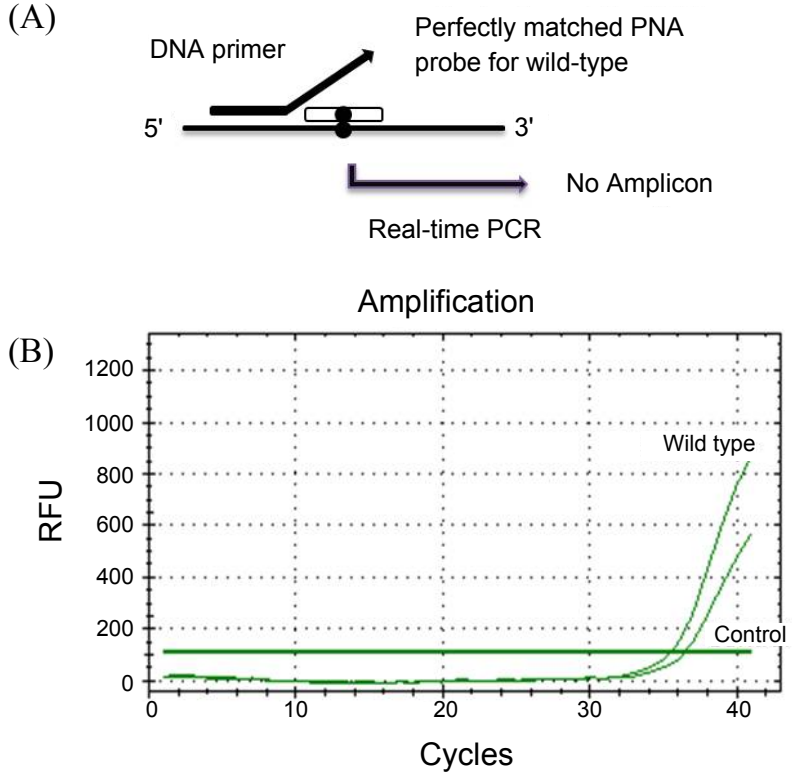

(C)
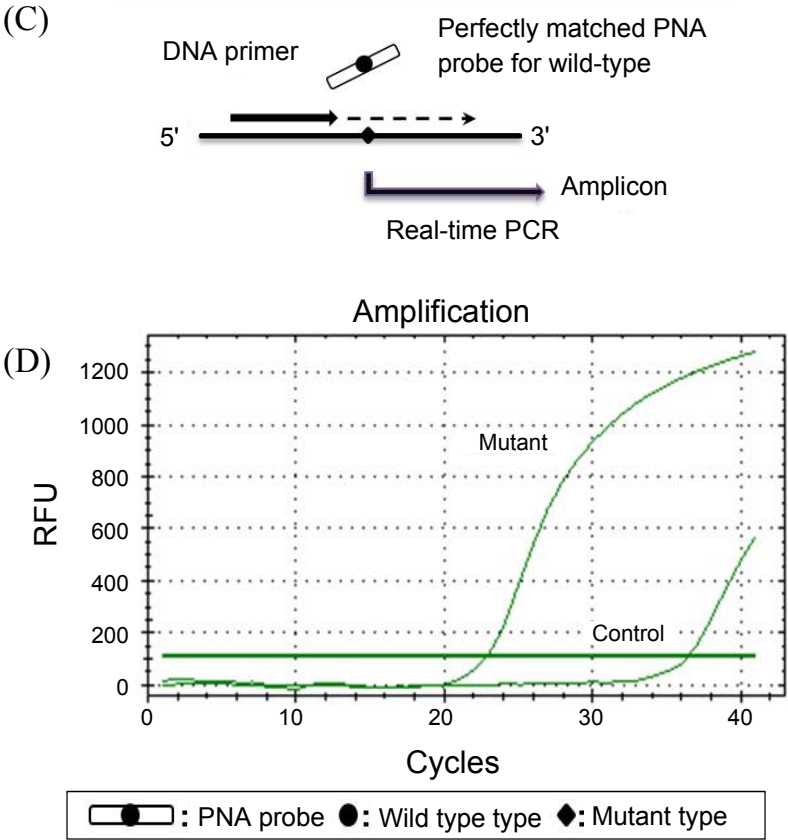

Figure 1. The PNA-mediated real-time PCR clamping system. The PNA oligomer was designed to bind to the bottom strand of the wildtype sequence, spanning mutational hotspots in exons $19-21$ of the EGFR gene. The forward PCR primer partially overlapped the PNA binding site. (A) During amplification, a PNA/DNA hybrid with a perfect match suppressed annealing of the PCR primer and amplification of wild-type DNA. (B) and (D) The detection signal was obtained by intercalation of SYBRGreen fluorescent dye of real-time PCR. (C) A PNA/DNA hybrid with a single base-pair mismatch did not suppress annealing of the PCR primer and amplification of mutant alleles.

$70{ }^{\circ} \mathrm{C}$ PNA annealing temperature that resulted the lowest $\mathrm{Ct}$ value (21.9) in mutant-type and the highest $\mathrm{Ct}$ value (38.3) in wild-type (Fig. 2B). For the PNA PCR clamping reaction, there seemed to be an optimal $63{ }^{\circ} \mathrm{C}$ primer annealing temperature that resulted the best compromise of $\mathrm{Ct}$ value (Fig. $2 \mathrm{C}$ ). This optimum primer annealing temperature $\left(63^{\circ} \mathrm{C}\right)$ produced the greatest selectivity such as high $\mathrm{Ct}$ value in wild-type and 
(A)

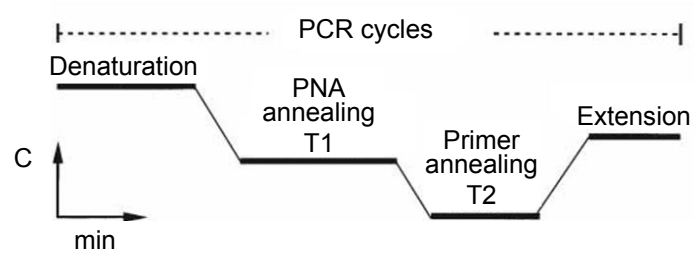

(B)
0
$\frac{0}{0}$
00
$\frac{0}{0}$
$\frac{0}{5}$
$\frac{0}{5}$
1

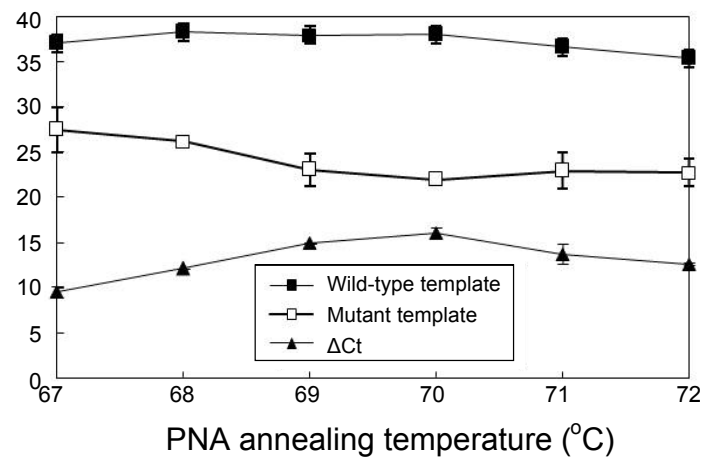

(C)
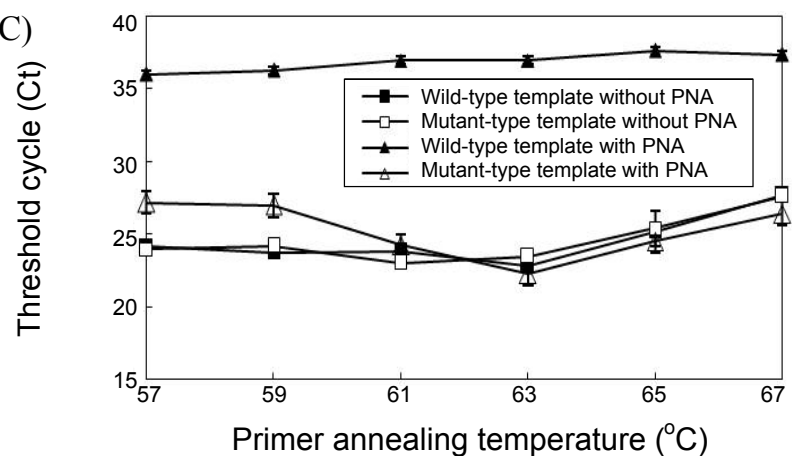

Figure 2. Optimization annealing temperature of clamping PNA and primer. (A) Schematic representation of the PCR cycle profile used in PNAmediated real-time PCR clamping system. T1: temperature of PNA annealing step $(20 \mathrm{sec})$ T2: temperature of primer annealing step. (B) Optimization of T1 for efficient PNA-mediated real-time PCR clamping. PNA was used to assay either a wild type or mutant DNA template. (C) Optimization of T2 for efficient PNA-mediated real-time PCR clamping. The values of Ct were represented for detection of T790M EGFR mutant. PCR primer was used to assay either a wild type or mutant DNA template.

(A) Exon 19 deletion
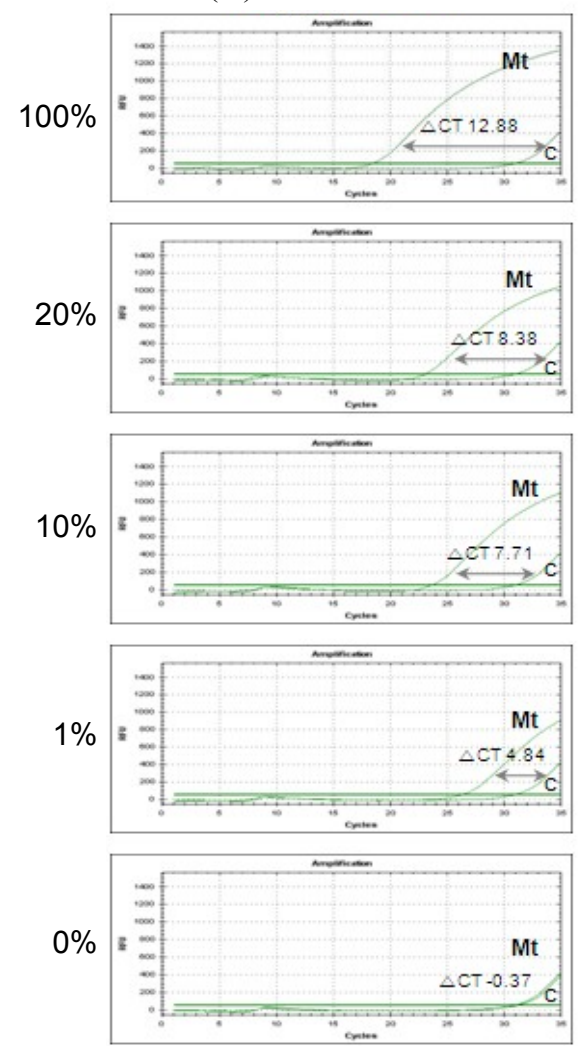

(B) T790M mutant
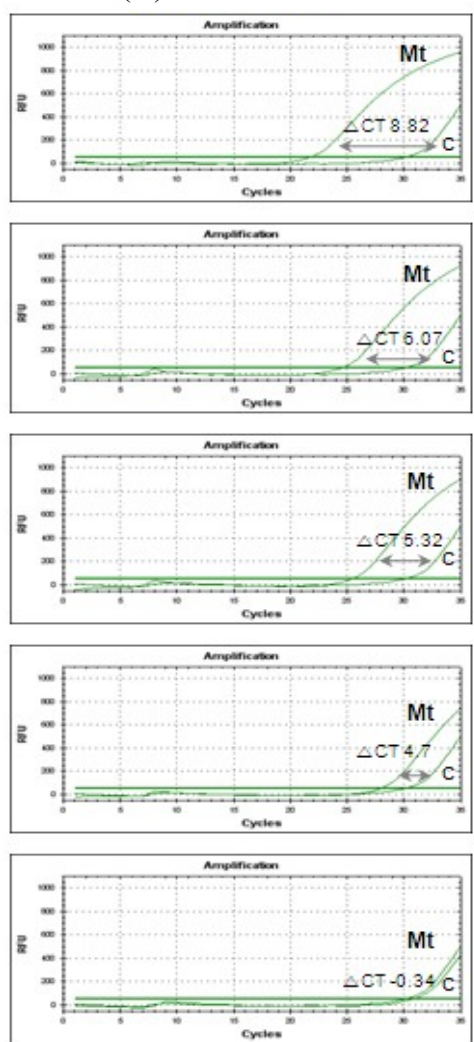

(C) L858R mutant
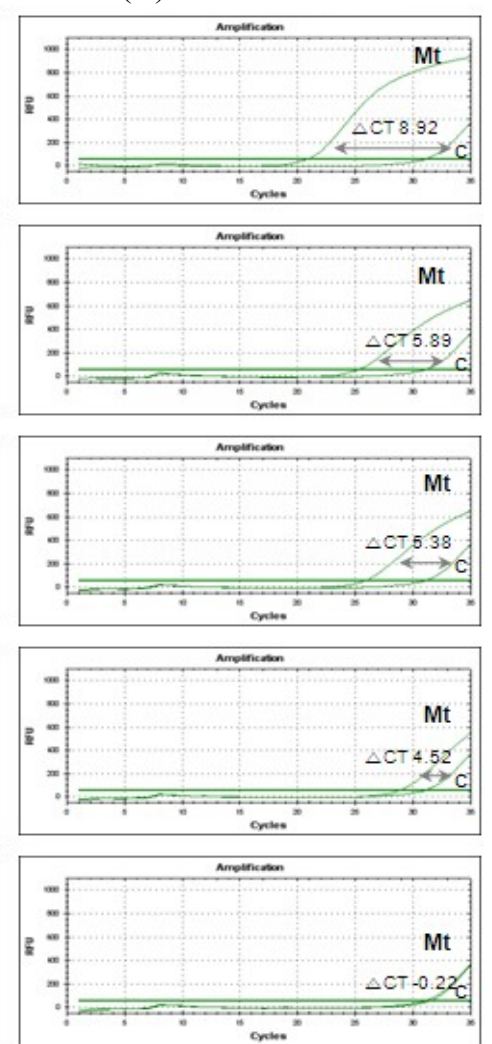

C : control sample, $\mathbf{M t}$ : mutant sample, $\triangle \mathbf{C t}=$ Control Ct values- Sample Ct values

Figure 3. Evaluation of the sensitivity of PNA-mediated real-time PCR for detection of EGFR mutations. Serially diluted standard templates were amplified by PNA-mediated real-time PCR clamping to detect EGFR mutations. Fifty nanogram amounts of genomic DNA were used as template in each method. Genomic DNA harboring (A) the 19deleltion mutation (HCC827GR), (B) the T790M (H1975) and (C) the L858R mutation (H1975) were serially diluted with wild-type DNA (from A549 cells). The proportions of mutant DNA were adjusted to $100 \%, 20 \%, 10 \%, 1 \%$, and $0 \%$. (mutant-type: wild-type). 
Table 2. Evaluation of the reproducibility of PNA-mediated real-time PCR

\begin{tabular}{|c|c|c|c|c|c|c|c|}
\hline \multirow{2}{*}{ Target } & \multirow{2}{*}{ Result } & \multicolumn{6}{|c|}{ Mutant concentration } \\
\hline & & $100 \%$ & $20 \%$ & $5 \%$ & $1 \%$ & $0.5 \%$ & $0 \%$ \\
\hline 19 del & Ct value & 19.3 & 21.6 & 23.3 & 25.3 & 29.3 & 31.8 \\
\hline Inter assay variation & $\mathrm{CV}(\%)$ & 1.7 & 1.2 & 1.4 & 1.6 & 1.6 & 1.3 \\
\hline $\mathrm{T} 790 \mathrm{M}$ & Ct value & 22.1 & 25.2 & 27.7 & 28.7 & 29.4 & 31.1 \\
\hline Inter assay variation & CV (\%) & 1.7 & 1.2 & 1.4 & 1.6 & 1.6 & 1.3 \\
\hline L858R & $\mathrm{Ct}$ value & 23.2 & 25.8 & 27.6 & 29.7 & 30.3 & 32.8 \\
\hline Inter assay variation & $\mathrm{CV}(\%)$ & 0.2 & 0.6 & 1.1 & 1.4 & 1.0 & 1.6 \\
\hline
\end{tabular}

Mean Ct (Threshold cycles) value calculated from three individual replicates on three separate lots. Inter-assay variation was calculated by comparing variation in $\mathrm{Ct}$ value on experiments on nine replicates. Coefficients of variation $(\mathrm{CV})$ represented the standard deviation divided by the mean. Genomic DNA harboring a homozygous 19del, T790M and L858R mutation (from H1975 and HCC827GR cells) was serially diluted with wild-type DNA (from A549 cells). The proportions of mutant DNA in samples were $100 \%, 20 \%, 5 \%, 1 \%, 0.5 \%$, and $0 \%$.

low $\mathrm{Ct}$ value in mutant-type with PNA, without measureable losses in sensitivity (low Ct value in wild and mutant type without PNA). The optimal annealing temperature of PNA and primer seem to be $70{ }^{\circ} \mathrm{C}$ and $63{ }^{\circ} \mathrm{C}$, respectively. These PCR conditions were used to discriminate single nucleotide mismatches on PNA mediated real-time PCR clamping.

Sensitivity and specificity of PNA-mediated real-time PCR clamping. To test specificity, various amounts of a wild-type plasmid (at $10^{2}-10^{3}$-fold excess) were mixed with a different plasmid containing each of the EGFR mutations. No false-positive or false-negative results were obtained (data not shown). Also, we evaluated assay sensitivity using DNAs extracted from cell lines harboring EGFR mutations. The results of PNAmediated real-time PCR clamping approaches used to detect EGFR mutant DNA diluted with wild-type DNA are shown in Figure 3. For analysis of exons 19, 20, and 21, the proportions of mutant DNA (from HCC827GR and H1975 cells) to normal DNA (from A549 cells), reflecting the extent of tumor cellularity, were adjusted to each of $100 \%, 20 \%, 10 \%, 1 \%$, and $0 \%$. Fifty nanogram DNA samples of genomic DNA were used as a template for PNA clamp. We were performed PNA clamp to determine the presence of T790M (exon 20), L858R (exon 21) point mutations and deletion (exon 19). The assay could detect mutations in the presence of up to a 100-fold excess (tumor cellularity $1 \%$ ) of the wild-type EGFR sequence, and showed a reverse correlation with the level of cellularity (Fig. 3).

Reproducibility of PNA-mediated real-time PCR clamping. To estimate the reproducibility of the PNA clamp method, wildtype cell line mixed mutation-bearing cellular DNA (wild-type $25 \mathrm{ng}$ amounts) were amplified; each assay was performed in triplicate of three lots $(n=9)$. Genomic DNA harboring a homozygous or heterozygous 19del, T790M and L858R mutation (from HCC827GR; 19del and cells H1975; T790M, L858R) was serially diluted with wild-type DNA (from A549 cells). The proportions of mutant DNA in samples were: $100 \%, 20 \%$, $5 \%, 1 \%, 0.5 \%$, and $0 \%$. Amplification profiles and $\mathrm{Ct}$ values (threshold cycle numbers) of the assay were compared between runs (Table 2). The intra- and inter-run coefficients of variance (in percentages) of the $\mathrm{Ct}$ values were calculated to be 1.7, 1.2, 1.4, 1.6, 1.6, and 1.3 for 19del samples, respectively; 1.7, 1.2, $1.4,1.6,1.6$, and 1.3 for T790M samples respectively; and 0.2 , $0.6,1.1,1.4,1.0$, and 1.6 for L858R of tumor cellularities from
$100 \%, 20 \%, 5 \%, 1 \%, 0.5 \%$, and $0 \%$ respectively. These results showed highly reproducible detection of EGFR mutation even at a concentration of $1 \%, 100$-fold excess amount of wild-type DNA.

\section{Discussion}

Mutation detection play a key role in the diagnostics, treatment, and prognosis assessment of cancer patient. ${ }^{12}$ Therefore, in the present study, we developed a rapid and sensitive PNAmediated real-time PCR clamping, to detect EGFR mutations. PNA-mediated real-time PCR clamping relies on the following three unique properties of PNA probes. First, PNA-DNA duplexes usually show a greater thermal stability than do the corresponding DNA-DNA duplexes, second, PNA oligomers can discriminate single nucleotide mismatches unambiguously than the corresponding DNA and, third, PNA oligomers are not recognized by DNA polymerases and can thus serve as sequenceselective clamps during PCR amplification. ${ }^{10}$ These characteristics mean that a PNA oligomer is a superior clamp probe when used to inhibit PCR amplification of wild-type sequence. ${ }^{11}$

In sensitivity evaluations, the PNA-mediated clamping method detected as little as $1 \%$ of a mutant allele in a mixture of mutant and wild-type DNA and no nonspecific amplifications (which often reduce sequencing sensitivity by elevating background noise) were apparent (Fig. 3). The amount of DNA template required was 10 - $50 \mathrm{ng}$ per reaction. This method will be applicable to detection of other known point mutations or small deletions, and is both rapid and highly sensitive, being particularly useful in the detection of low-level mutant DNAs in the presence of a high-level of wild-type DNAs. And we confirmed that this assay enabled simple, sensitive and reproducible detection of mutant DNA in the presence of a 100-fold excess of wild-type DNA, using 50 ng DNA as template in three tests, each performed in triplicate $(n=9)$ (Table 2). Highly reproducible results were obtained in the presence of as little as $1 \%$ of the mutant DNA, using low DNA levels (10 ng amounts) from mutant cell lines. We have thus established PNA-mediated clamping platform technology for detection of EGFR mutations. Using this approach, we detected small amounts of mutant DNA that were not able to detect by direct sequencing, and identified patients for whom this assay provided clinical infor- 
mation that was unavailable from other tests; such data may contribute to therapeutic decisions.

\section{Conclusion}

Our PNA-mediated real-time PCR clamping assay is rapid and sensitive method for detection of low-level EGFR mutations and become a powerful tool for detecting low-level mutation in clinical samples.

Acknowledgments. This work was supported by the Small and Medium Business Administration supported by the Korean Government (Grant No. S1060400).

\section{References}

1. Inoue, A.; Kobayashi, K.; Usui, K.; Maemondo, M.; Okinaga, S.; Mikami, I.; Ando, M.; Yamazaki, K.; Saijo, Y.; Gemma, A.; Miyazawa, H.; Tanaka, T.; Ikebuchi, K.; Nukiwa, T.; Morita, S.; Hagiwara, K. J Clin Oncol. 2009, 27, 1394.

2. Yamamoto, H.; Toyooka, S.; Mitsudomi, T. Lung Cancer 2009, $63,315$.

3. Onitsuka, T.; Uramoto, H.; Naohiro, N.; Takenoyama, M.; Hanagiri, T.; Sugio, K.; Yasumoto, K. Lung Cancer 2010, 68, 198.

4. Yung, T. K.; Chan, K. C.; Mok, T. S.; Tong, J.; To, K. F.; Lo, Y.
M. Clin. Cancer. Res. 2009, 15, 2076.

5. Sequist, L. V.; Engelman, J. A.; Lynch, T. J. J. Clin. Oncol. 2009, 27, 2589.

6. Park, K.; Jung, W.; Choi, B. R.; Park, H.; Kim, D. E. Bull. Korean Chem. Soc. 2010, 31, 7.

7. Maemondo, M.; Inoue, A.; Kobayashi, K.; Sugawara, S.; Oizumi, S.; Isobe, H.; Gemma, A.; Harada, M.; Yoshizawa, H.; Kinoshita, I.; Fujita, Y.; Okinaga, S.; Hirano, H.; Yoshimori, K.; Harada, T.; Ogura, T.; Ando, M.; Miyazawa, H.; Tanaka, T.; Saijo, Y.; Hagiwara, K.; Morita, S.; Nukiwa, T.; North-East Japan Study Group. N. Engl. J. Med. 2010, 362, 2380.

8. Brevet, M.; Arcila, M.; Ladanyi, M. J. Mol. Diagn. 2010, 12, 169.

9. Maheswaran, S.; Sequist, L. V.; Nagrath, S.; Ulkus, L.; Brannigan, B.; Collura, C. V.; Inserra, E.; Diederichs, S.; Iafrate, A. J.; Bell, D. W.; Digumarthy, S.; Muzikansky, A.; Irimia, D.; Settleman, J.; Tompkins, R. G.; Lynch, T. J.; Toner, M.; Haber, D. A. N. Engl. J. Med. 2008, 359, 366.

10. Marchetti, A.; Milella, M.; Felicioni, L.; Cappuzzo, F.; Irtelli, L.; Del Grammastro, M.; Sciarrotta, M.; Malatesta, S.; Nuzzo, C.; Finocchiaro, G.; Perrucci, B.; Carlone, D.; Gelibter, A. J.; Ceribelli, A.; Mezzetti, A.; Iacobelli, S.; Cognetti, F.; Buttitta, F. Neoplasia. 2009, 11, 1084 .

11. Miyazawa, H.; Tanaka, T.; Nagai, Y.; Matsuoka, M.; Huqun,; Sutani, A.; Udagawa, K.; Zhang, J.; Hirama, T.; Murayama, Y.; Koyama, N.; Ikebuchi, K.; Nagata, M.; Kanazawa, M.; Nukiwa, T.; Takenoshita, S.; Kobayashi, K.; Hagiwara, K. Cancer Sci. 2008, 99 , 595.

12. Carlo, M.; Croce, M. N. Engl. J. Med. 2008, 358, 502-511. 